\title{
Comparison of the Predictive Roles of Risk Scores of In-Hospital Major Adverse Cardiovascular Events in Patients with Non-ST Elevation Myocardial Infarction Undergoing Percutaneous Coronary Intervention
}

\author{
Erdal Aktürk Lütfü Aşkın Hakan Taşolar Serdar Türkmen Hakan Kaya \\ Department of Cardiology, Adiyaman University, Adiyaman, Turkey
}

\section{Significance of the Study}

- This study examines the relationship between risk scores and major adverse cardiovascular events (MACE) in patients with non-ST elevation myocardial infarction undergoing percutaneous coronary intervention. All risk scores were able to predict MACE, but the SYNTAX score II (SS-II) was also able to predict in-hospital mortality, nonfatal myocardial infarction, and stent thrombosis. The SS-II scoring system appears to be the most comprehensive scoring system as it is angiographic and has 6 clinical variables.

\section{Keywords}

Risk scores · Cardiovascular events · Non-ST elevation myocardial infarction

\begin{abstract}
Objective: We evaluated the relationship between various risk scores (SYNTAX score [SS], SYNTAX score-II [SS-II], thrombolysis in myocardial infarction [TIMI] risk scores, and Global Registry of Acute Coronary Events [GRACE] risk scores) and major adverse cardiovascular events (MACE) in non-ST elevation myocardial infarction (NSTEMI) patients undergoing percutaneous coronary intervention (PCI). Subjects and Methods: The study population were selected from among 589 patients who underwent coronary angiography with a
\end{abstract}

\begin{tabular}{ll}
\hline KARGER & $\begin{array}{l}\text { (c) } 2018 \text { The Author(s) } \\
\text { Published by S. Karger AG, Basel Openger }\end{array}$ \\
E-Mail karger@karger.com & $\begin{array}{l}\text { This is an Open Access article licensed under the Creative Commons } \\
\text { Attribution-NonCommercial-4.0 International License (CC BY-NC) } \\
\text { (http://www.karger.com/Services/OpenAccessLicense), applicable to } \\
\text { the online version of the article only. Usage and distribution for } \\
\text { commercial purposes requires written permission. }\end{array}$
\end{tabular}

diagnosis of NSTEMI. TIMI and GRACE risk scores were calculated. SS and SS-II were calculated in all patients, and points were added according to the predefined algorithm, taking into account the other 6 clinical variables being monitored (age, sex, left ventricular ejection fraction, creatinine clearance, chronic obstructive pulmonary disease, and peripheral artery disease). Patients were classified into tertile 1 (SS $<22$ ), tertile 2 (SS 23-32), and tertile 3 (SS > 32). Results: The group with high SS-II for $\mathrm{PCl}$ values in the risk scores were observed from tertile 1 to tertile 3 (from $25.0 \pm 7.7$ to $31.6 \pm 9.4, p<$ 0.001 , respectively). The SS-II score in patients with $\mathrm{PCI}$ was an independent predictor of MACE, in-hospital mortality, nonfatal myocardial infarction, and stent thrombosis (OR $1.082,95 \% \mathrm{Cl} 1.036-1.131, p<0.001)$. The overall MACE, inhospital mortality, and nonfatal myocardial infarction rates
Erdal Aktürk, MD

Department of Cardiology

Adryaman University

Adıyaman (Turkey)

E-Mail erdalakturk@hotmail.com 
were significantly higher in the high SS-II for $\mathrm{PCl}$ group $(p<$ 0.001). Conclusion: TIMI and GRACE risk scores were able to predict MACE. In addition to these, SS-II was also able to predict in-hospital mortality, nonfatal myocardial infarction, and stent thrombosis.

(c) 2018 The Author(s)

Published by S. Karger AG, Basel

\section{Introduction}

Patients hospitalized for non-ST elevation myocardial infarction (NSTEMI) vary widely in terms of the severity of the disease based on clinical and laboratory characteristics [1]. Coronary atherosclerosis is the main cause of NSTEMI. Risk stratification is essential for adequate clinical decision making. The risk of morbidity and mortality in NSTEMI patients varies according to the presence of initial risk factors, clinical syndrome characteristics, and management strategy [2]. Cardiologists use risk scoring to determine the severity and complexity of coronary artery disease (CAD) in patients with acute coronary syndrome (ACS) [2]. The most commonly used scores are the thrombolysis in myocardial infarction (TIMI) risk score, the Global Registry of Acute Coronary Events (GRACE) score, and the synergy between percutaneous coronary intervention (PCI) with taxus and cardiac surgery (SYNTAX) score (SS). The prognostic values of these scores have been validated in multiple clinical trials $[1,3,4]$. However, the absence of clinical variables in SS has been identified as a significant limitation when applied to patients with complex CAD in the right stratification capacity. To address these limitations, SS-II was recently developed [5]. SS-II has been associated with both angiographic (anatomic SS) and clinical variables, which include age, gender, left ventricular ejection fraction, creatinine clearance, chronic obstructive pulmonary disease, and peripheral vascular disease [6]. It provides a more accurate and individualized estimate of mortality, and thus leads to a clinically more useful tool for bedside decision making and management of complex CAD [7].

SS-II is used to estimate 4-year mortality for patients scheduled for myocardial revascularization (coronary bypass or PCI) [8]. Therefore, the aim of this study was to compare the relative values of clinical risk assessments and scoring systems in predicting the extent of CAD and to assess the accuracy of SS-II in determining major adverse cardiovascular events (MACE) in NSTEMI patients.

\section{Subjects and Methods}

\section{Study Design}

This was an observational, cross-sectional study performed at our training and research hospital. This study complied with the Declaration of Helsinki and was approved by the local ethics committee. Informed consent was obtained from all participants. The study population were selected from among 589 patients who underwent coronary angiography with a diagnosis of NSTEMI. After the exclusion criteria were applied, 573 eligible consecutive patients, who were admitted to the coronary care unit of our institution between January 2015 and January 2017, were selected. NSTEMI was defined as new-onset or worsening chest pain occurring at rest or with minimal exertion, with an elevation of cardiac troponin values with at least 1 value above the 99th percentile upper reference limit for the previous $48 \mathrm{~h}$. Atypical chest pain was assessed as follows: a duration more than $20 \mathrm{~min}$, new-onset angina, and an increase in the frequency, duration, and severity of pain. The cardiac troponin was evaluated as a positive biomarker with a threshold of $0.04 \mathrm{ng} / \mathrm{mL}$, as measured by the Alere Triage MeterPro device (Alere Inc., San Diego, CA, USA). All patients selected had undergone coronary angiography during hospitalization. SS analysis is only valid for natural coronary arteries, so patients with previous coronary artery bypass grafts were excluded [9]. Patients with cardiogenic shock were also excluded from the study. Intrastent restenosis was also scored as de novo lesions.

\section{Study Protocol}

The medical records of all patients were examined for data regarding $\mathrm{CAD}$, including medical history and history of previous myocardial infarction, hypertension, diabetes mellitus, dyslipidemia or smoking, family history of chronic heart failure, previous ischemic stroke, transient ischemic attacks or peripheral artery disease, presenting symptoms, biochemical and electrocardiography findings, echocardiographic examinations, and digital or nondigital hospital outcomes. Hypertension was defined as receiving antihypertensive therapy and/or having an arterial blood pressure of $>140 / 90$ in more than one determination. Diabetes mellitus was defined as a fasting blood glucose of $\geq 126 \mathrm{mg} / \mathrm{dL}$ or the current use of antidiabetic medications. Hyperlipidemia was noted when the patient had a total cholesterol of $>200 \mathrm{mg} / \mathrm{dL}$, had a triglyceride level of $>150 \mathrm{mg} / \mathrm{dL}$, and had a history of dyslipidemia and/or was undergoing antilipidemic therapy. Active smokers or patients with a smoking history of at least 1 pack/year until 1 month before study inclusion were considered to have a smoking history. A family history of chronic heart failure was defined as sudden cardiac death in a male first-degree relative aged under 55 years or in a female first-degree relative aged under 65 years. M-mode echocardiography was used to measure left ventricular ejection fraction in the two-dimensional echocardiographic apical 4-chamber view. Body mass index was calculated as weight in kilograms divided by the square of the height in meters. Vascular disease was considered to be the presence of peripheral artery disease.

Selective coronary angiography was performed by the femoral approach, using the standard Judkins technique at a rate of 30 frames/s in multiple, angulated views (Allura Xper FD10; Philips Healthcare, Best, The Netherlands). Coronary angiograms were assessed independently by 2 invasive cardiologists, who were blinded to the clinical findings. Significant vessel disease was de- 
Table 1. Baseline demographic and clinical parameters of the study population

\begin{tabular}{|c|c|c|c|c|c|c|}
\hline Variables & $\begin{array}{l}\text { Tertile } 1 \\
\text { SS <23 } \\
(n=334)\end{array}$ & $\begin{array}{l}\text { Tertile } 2 \\
\text { SS } 23-32 \\
(n=170)\end{array}$ & $\begin{array}{l}\text { Tertile } 3 \\
\mathrm{SS}>32 \\
(n=69)\end{array}$ & $\begin{array}{l}p \\
(\mathrm{~T} 1-\mathrm{T} 2)\end{array}$ & $\begin{array}{l}p \\
(\mathrm{~T} 1-\mathrm{T} 3)\end{array}$ & $\begin{array}{l}p \\
(\mathrm{~T} 2-\mathrm{T} 3)\end{array}$ \\
\hline Male gender, \% & 40.3 & 22.9 & 8.9 & 0.606 & 0.434 & 0.606 \\
\hline $\mathrm{DM}, \%$ & 4 & 2.3 & 1.0 & 0.786 & 0.597 & 0.786 \\
\hline COPD, $\%$ & 2.3 & 1.6 & 0.2 & 0.179 & 0.314 & 0.179 \\
\hline $\mathrm{PAD}, \%$ & 3.7 & 1.4 & 1.0 & 0.235 & 0.467 & 0.235 \\
\hline Previous PCI history, \% & 15.7 & 7.3 & 4.0 & 0.175 & 0.316 & 0.175 \\
\hline Family history, \% & 17.7 & 5.9 & 3.5 & 0.133 & 0.825 & 0.133 \\
\hline BMI & $26.2 \pm 4.0$ & $25.9 \pm 3.5$ & $24.9 \pm 4.2$ & 0.457 & 0.020 & 0.054 \\
\hline SS & $12.6 \pm 5.2$ & $26.3 \pm 1.9$ & $34.8 \pm 4.5$ & $<0.001$ & $<0.001$ & $<0.001$ \\
\hline LVEF, \% & $58.6 \pm 3.4$ & $58.0 \pm 3.3$ & $57.4 \pm 3.1$ & 0.069 & 0.008 & 0.191 \\
\hline GLU, mg/dL & $115.5 \pm 54.3$ & $116.9 \pm 53.0$ & $125.5 \pm 68.4$ & 0.774 & 0.258 & 0.356 \\
\hline Cre, mg/dL & $0.82 \pm 0.2$ & $0.82 \pm 0.2$ & $0.80 \pm 0.2$ & 0.806 & 0.500 & 0.588 \\
\hline $\mathrm{CCr}, \mathrm{mL} / \mathrm{min}$ & $106.2 \pm 55.8$ & $105.9 \pm 49.4$ & $101.5 \pm 44.8$ & 0.962 & 0.512 & 0.516 \\
\hline $\mathrm{TC}, \mathrm{mg} / \mathrm{dL}$ & $171.8 \pm 39.0$ & $173.6 \pm 36.9$ & $167.7 \pm 36.5$ & 0.608 & 0.422 & 0.258 \\
\hline $\mathrm{TG}, \mathrm{mg} / \mathrm{dL}$ & $152.8 \pm 74.2$ & $151.7 \pm 74.8$ & $141.8 \pm 61.8$ & 0.876 & 0.247 & 0.328 \\
\hline $\mathrm{HDL}, \mathrm{mg} / \mathrm{dL}$ & $32.1 \pm 8.3$ & $31.9 \pm 8.1$ & $33.3 \pm 9.2$ & 0.843 & 0.270 & 0.250 \\
\hline $\mathrm{LDL}, \mathrm{mg} / \mathrm{dL}$ & $106.4 \pm 27.9$ & $104.2 \pm 33.7$ & $110.7 \pm 25.7$ & 0.442 & 0.233 & 0.149 \\
\hline Total protein, g/dL & $6.4 \pm 0.6$ & $6.4 \pm 0.5$ & $6.3 \pm 0.5$ & 0.553 & 0.632 & 0.385 \\
\hline Albumin, g/dL & $3.4 \pm 0.4$ & $3.4 \pm 0.3$ & $3.4 \pm 0.3$ & 0.764 & 0.405 & 0.650 \\
\hline AST, U/L & $30.3 \pm 18.5$ & $31.2 \pm 21.8$ & $31.6 \pm 21.0$ & 0.623 & 0.607 & 0.903 \\
\hline ALT, U/L & $27.6 \pm 19.0$ & $28.2 \pm 16.7$ & $31.3 \pm 25.9$ & 0.754 & 0.273 & 0.362 \\
\hline
\end{tabular}

One-way ANOVA and $\chi^{2}$ tests were used. ALT, alanine transaminase; AST, aspartate transaminase; BMI, body mass index; SS, SYNTAX score; CABG, coronary artery bypass graft; COPD, chronic obstructive pulmonary disease; Cre, creatinine; CCr, creatinine clearance; DM, diabetes mellitus; GLU, glucose; HT, hypertension; Na, sodium; K, potassium; LVEF, left ventricular ejection fraction; PAD, peripheral arterial disease; PCI, percutaneous coronary intervention; TC, total cholesterol; TG, triglyceride; HDL, high-density lipoprotein; LDL, low-density lipoprotein; WBC, white blood cell; Hb, hemoglobin; PLT, platelet count.

fined as the presence of $\geq 50 \%$ luminal diameter stenosis in at least 1 major coronary artery. Each coronary lesion with a diameter stenosis $\geq 50 \%$ in vessels $\geq 1.5 \mathrm{~mm}$ was required to be scored. The interventional cardiologists calculated the numeric values of the SS, and tertiles $(\leq 22,>22-32,>32)$ of the score were used. SS-II (for both PCI and coronary bypass) was derived using the basic clinical features of patients as previously described [6]. The 2 blinded cardiologists calculated the TIMI risk score by allocating 1 point each for having peripheral artery disease, being $\geq 65$ years, having $\geq 3$ CAD risk factors, having known CAD (stenosis $\geq 50 \%$ ), having used acetylsalicylic acid in the previous 7 days, having severe angina ( $\geq 2$ episodes in $24 \mathrm{~h}$ ), having a positive cardiac mark- er and the presence of electrocardiography ST changes $\geq 0.5 \mathrm{~mm}$. In addition, the GRACE risk score was calculated by allocating 1 point each for age, heart rate/pulse, systolic blood pressure, creatinine, cardiac arrest at admission, the presence of ST segment deviation on electrocardiography, elevated/abnormal cardiac enzymes, and Killip classification (signs/symptoms). Briefly, the initial SS was calculated, and points were added according to the predefined algorithm, taking into account the other 6 clinical variables (age, sex, left ventricular ejection fraction, creatinine clearance, chronic obstructive pulmonary disease, and peripheral artery disease). Patients were classified and compared according to the tertiles of their SS. 
Fig. 1. Comparison of ROC curves of the SS-II score, TIMI, and GRACE scores in the risk stratification of in-hospital MACE in NSTE-ACS patients. CABG, coronary artery bypass graft.

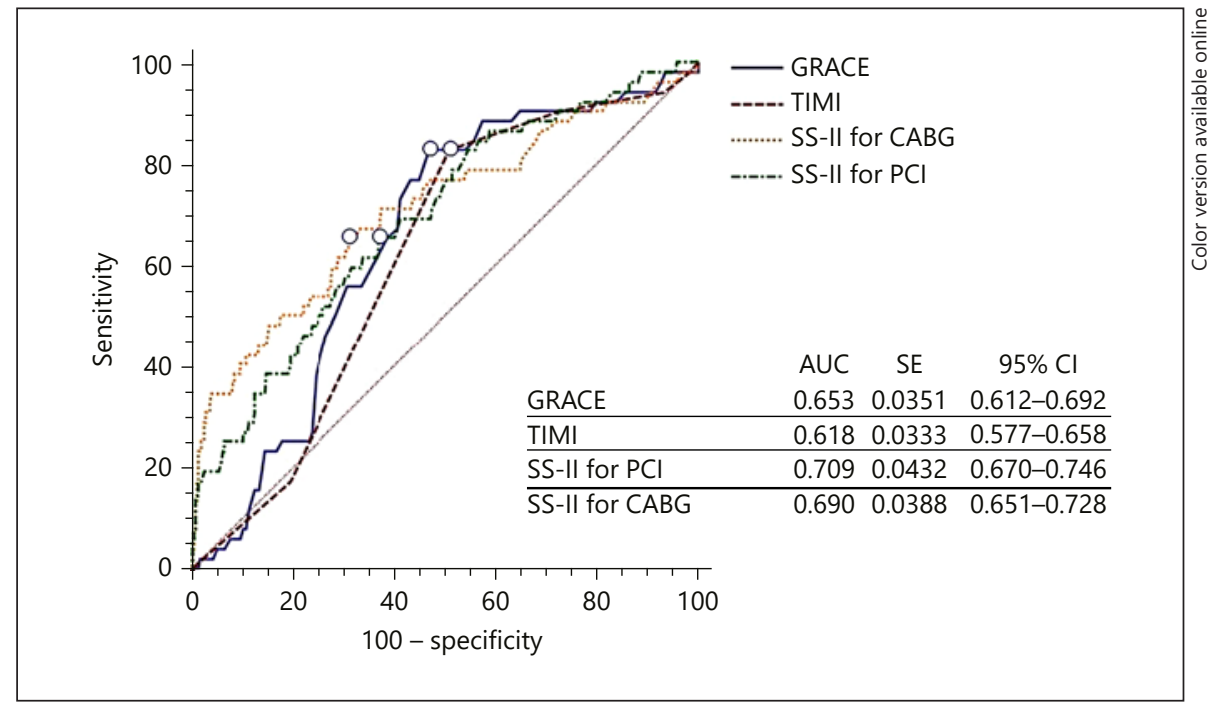

Table 2. Independent predictors of MACE: logistic regression analysis

\begin{tabular}{llc}
\hline Variables & Multivariate OR (95\% CI) & Multivariate $p$ \\
\hline SS & $0.915(0.873-0.959)$ & $<0.001$ \\
TIMI score & $0.567(0.346-0.931)$ & 0.025 \\
GRACE score & $0.966(0.942-0.991)$ & 0.007 \\
SS-II for PCI & $1.146(1.085-1.211)$ & $<0.001$ \\
SS-II for CABG & $1.053(1.017-1.090)$ & 0.004 \\
\hline
\end{tabular}

SS, SYNTAX score; CABG, coronary artery bypass graft; PCI, percutaneous coronary intervention.

In-hospital MACE components included nonfatal myocardial infarction, in-hospital mortality, and stent thrombosis before discharge. Nonfatal myocardial infarction was defined as persistent chest pain during in-hospital follow-up and new electrocardiography changes recorded at the same time as elevated values of $\geq 20 \%$ in cardiac markers. Stent thrombosis was defined based on academic research [7]. In-hospital mortality was defined as death due to myocardial infarction or other cardiac causes and in-hospital cardiac arrests.

\section{Statistical Analysis}

Statistical Package for the Social Sciences (SPSS) software (version 21.0 for Windows; SPSS Inc., Chicago, IL, USA) was used for all statistical calculations. The Kolmogorov-Smirnov test was used to confirm whether the data fit a normal distribution. Continuous variables were presented as means \pm standard deviation (SD) and/or medians (minimum to maximum). Categorical variables were expressed as the number and proportions, while continuous variables were expressed as the mean \pm SD. A one-way analysis of variance and the Kruskal-Wallis test were used to compare the 3 groups. Multivariate logistic regression analysis was used to identify clinical predictors of MACE, inhospital mortality, and stent thrombosis and nonfatal myocardial infarction. The receiver-operating characteristics (ROC) curve was used to test the predictive accuracy of the SS-II for PCI and coronary bypass with respect to the presence of high risk $(>22)$ or low risk $(<22)$, based on the SS. Pairwise comparisons of the ROC curves were used to check the in-hospital prognostic value of the SS-II for PCI and coronary bypass scores with the TIMI and GRACE risk scores. A $p$ value $<0.05$ was considered to indicate statistical significance.

\section{Results}

In this study, 573 patients with NSTEMI (mean age $62.3 \pm 12.5$ years, $72.1 \%$ male) were enrolled. The baseline demographic and clinical parameters of the study population according to SS tertile are shown in Table 1. SS showed a significant increase between tertile 1 and tertile 3 (from $12.6 \pm 5.2$ to $34.8 \pm 4.5, p<0.001$ ). SS-II for PCI showed a significant increase between tertiles (from 25.0 \pm 7.7 to $31.6 \pm 9.4, p<0.001$ ), as did SS-II for coronary bypass (from $10.6 \pm 11.2$ to $29.3 \pm 7.7, p<0.001$ ). The TIMI score showed a significant increase between tertiles (from $2.5 \pm 1.4$ to $3.7 \pm 1.0, p<0.001$ ), and GRACE score also showed a significant increase between tertiles (from $97.0 \pm 24.3$ to $115.4 \pm 24.9, p<0.001$ ). Smoking, hypertension, diabetes mellitus hyperlipidemia, chronic obstructive pulmonary disease, peripheral artery disease, previous PCI history, previous stroke, family history, and total cholesterol $(p<0.05)$ also significantly increased between 
Table 3. Comparison of the risk scores according to the number of diseased vessels

\begin{tabular}{lcccc}
\hline & $\begin{array}{l}\text { Tertile 1 } \\
(n=155)\end{array}$ & $\begin{array}{l}\text { Tertile 2 } \\
(n=215)\end{array}$ & $\begin{array}{l}\text { Tertile 3 } \\
(n=202)\end{array}$ & $\begin{array}{l}p \\
\text { value }\end{array}$ \\
\hline SS & $12.0 \pm 5.2$ & $15.2 \pm 6.7$ & $29.3 \pm 5.0$ & $<0.001$ \\
SS-II for PCI & $25.7 \pm 8.7$ & $25.7 \pm 8.2$ & $29.5 \pm 8.6$ & $<0.001$ \\
SS-II for CABG & $15.7 \pm 13.2$ & $8.6 \pm 8.7$ & $10.7 \pm 10.1$ & $<0.001$ \\
TIMI score & $1.9 \pm 0.5$ & $2.6 \pm 1.2$ & $3.0 \pm 1.2$ & $<0.001$ \\
GRACE score & $89.3 \pm 15.09$ & $103.5 \pm 24.2$ & $111.9 \pm 23.7$ & $<0.001$ \\
\hline
\end{tabular}

One-way analysis of variance was used to compare the 3 groups.

Table 4. In-hospital adverse events

\begin{tabular}{lllllll}
\hline Variables & $\begin{array}{l}\text { Overall } \\
(n=573)\end{array}$ & $\begin{array}{l}\text { SS-II for } \\
\text { PCI }<27.9 \\
(n=272)\end{array}$ & $\begin{array}{l}\text { SS-II for } \\
\text { PCI } \geq 27.9 \\
(n=301)\end{array}$ & $p$ & $\begin{array}{l}\text { SS-II for } \\
\text { CABG }<6.45 \\
(n=285)\end{array}$ & $\begin{array}{l}\text { SS-II for } \\
\text { CABG } \geq 6.45 \\
(n=288)\end{array}$ \\
\hline MACE, \% & 7.4 & 3.1 & 6.3 & 0.029 & 3.0 & 6.5 \\
In-hospital mortality, \% & 1.7 & 0.0 & 1.7 & 0.002 & 0.0 & 0.011 \\
Nonfatal MI, \% & 3.5 & 1.0 & 2.4 & 0.111 & 1.0 & 2.7 \\
Stent thrombosis, \% & 5.6 & 2.6 & 3.0 & 0.945 & 2.4 & 3.3 \\
\hline
\end{tabular}

Categorical variables were compared with the $\chi^{2}$ or Fisher exact tests. PCI, percutaneous coronary intervention; CABG, coronary artery bypass graft; MACE, major adverse cardiovascular events; MI, myocardial infarction.

tertile 1 and tertile 3. By contrast, left ventricular ejection fraction significantly decreased between tertile 1 and tertile $3(p=0.01)$. Multivariate logistic regression analysis revealed that TIMI, GRACE, and SS-II for PCI were independent predictors of MACE (Table 2; OR 1.967, 95\% CI 1.475-2.622, $p<0.001$, OR 1.021, 95\% CI 1.004-1.038, $p=0.02$, and OR 1.082, 95\% CI 1.036-1.131, $p<0.001$, respectively).

In-hospital MACE is shown in Table 3. Overall, MACE, in-hospital mortality, and nonfatal myocardial infarction were significantly higher in the high SS-II for PCI group $(p<0.05)$. In addition to this, MACE, in-hospital mortality, and stent thrombosis were significantly higher in the high SS-II for coronary bypass group $(p<0.05)$. The pairwise comparisons of the ROC curves of these scores are shown in Table 4, with no significant differences detected in the AUC between both SS-II for PCI and coronary bypass. Figure 1 shows a comparison of ROC curves of the SS-II for PCI and SS-II for coronary bypass scores in the risk stratification of the in-hospital MACE in NSTE-ACS patients. The SS-II for PCI $>27.9$ value predicted in-hospital mortality with a sensitivity of $64.5 \%$ and specificity of $63.0 \%$ (AUC 0.690 ; 95\% CI 0.650-0.727, $p<0.001$; Fig. 2).

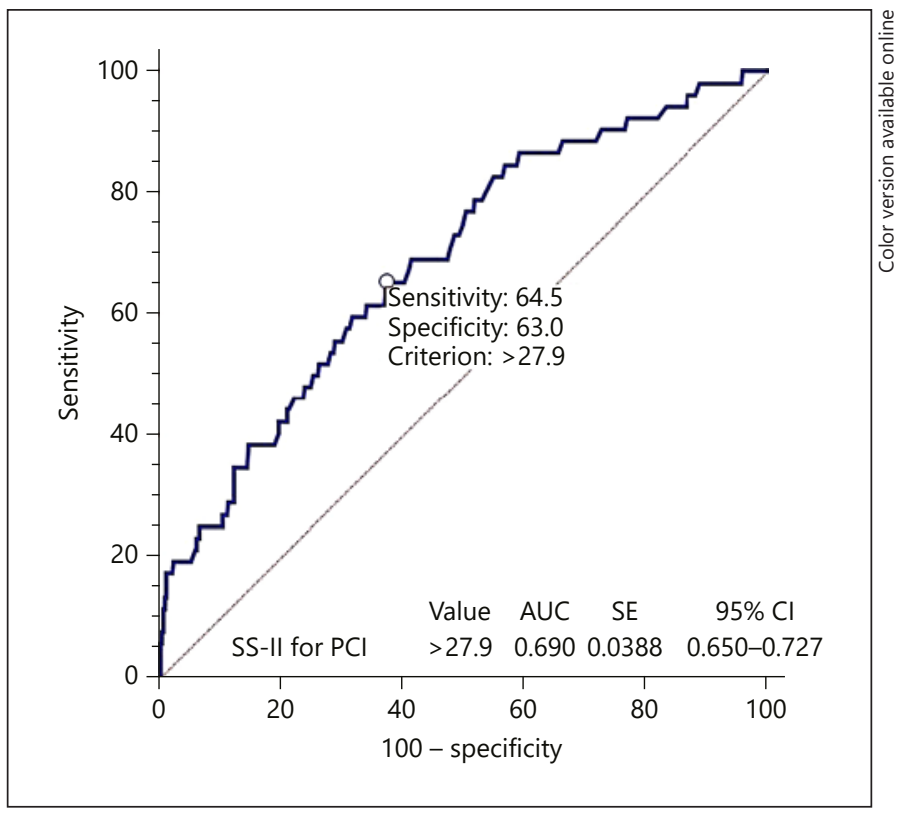

Fig. 2. The SS-II value for PCI $>27.9$ predicted in-hospital mortality with a sensitivity of $64.5 \%$ and specificity of $63.0 \%$. 


\section{Discussion}

The main findings of our study can be summarized as follows: (1) the SS-II for PCI, SS-II for coronary bypass, TIMI, and GRACE risk scores increased from tertile 1 to tertile 3 according to SS in patients with NSTEMI; (2) TIMI, GRACE, and SS-II for PCI scores in patients with NSTEMI were independent predictors of MACE, and SSII was also able to predict in-hospital mortality, nonfatal myocardial infarction, and stent thrombosis; (3) ROC comparisons showed that SS-II PCI was similar to other risk scores in the prediction and risk stratification of inhospital MACE in NSTEMI patients, and (4) a score of SSII PCI >27.9 may predict in-hospital mortality.

The GRACE score is a strong, independent predictor of MACE in patients with ACS [10]. It is widely accepted that the TIMI risk score is one of the most important scoring systems, preferred for risk stratification in patients with ACS, and is shown to be beneficial in many studies with large patient populations [11]. TIMI and GRACE scores are useful for the initial classification of NSTE-ACS patients, but they are not optimized for patients with PCI due to the lack of angiographic findings in these scoring systems. The SS has been developed as a comprehensive angiographic scoring tool for the quantification of coronary lesions with respect to their number, location, and complexity. It was initially tested in patients with stable $\mathrm{CAD}$, multi-vessel disease, or complex coronary lesions allocated to PCI or coronary bypass in the landmark SYNTAX trial [9]. Subsequently, this score was also validated for left main CAD and in an all-comers population undergoing PCI. It is a good predictor of adverse cardiovascular events, including cardiac death, myocardial infarction, and target lesion revascularization [9]. The TIMI, SS, and GRACE scoring systems are widely used in routine, clinical practice because of their proven value in large clinical trials; however, the SS-II scoring system is better able to predict clinical events. Because SS-II uses a combination of both angiographic (anatomic SS) and clinical variables, it has the ability to predict postprocedural outcomes that have a significant clinical impact, such as informing patients and families about adverse outcomes related to a given revascularization strategy [13]. In this study, the TIMI, GRACE, and SS-II for PCI were independent predictors of MACE, but SS-II was also able to predict in-hospital mortality, non-fatal myocardial infarction, and stent thrombosis.

The superiority of scoring systems created by combining anatomic and clinical variables over the anatomic SYNTAX has been pointed out previously [14]. With the unique approach of SS-II, a subset of patients with low
(23), intermediate (23-32) or high (>32) anatomical SS were defined objectively for coronary bypass or PCI, which would have predicted a lower, similar, or higher 4-year mortality. Significantly, these findings have been verified in the DELTA registry [15]. Girasis et al. [16] were the first investigators to show that the addition of patient age, creatinine, and ejection fraction to SYNTAX, to create clinical SYNTAX, significantly increased the predictability of events in PCI patients. SS-II predicted a 55.2\% probability that there was no statistically significant difference in mortality between the PCI and coronary bypass arms of the expanded clinical evaluation of lovastatin (EXCEL) at 4 years. This will likely be secondary to the clinical status of patients included in the EXCEL. Generally, the key exclusion criteria for participants in the EXCEL [8] were normal renal function, preserved left ventricular ejection fraction, being of male sex, and having significantly complex CAD (SS $\geq 33$ ). In the SYNTAX trial, being of female gender, with reduced left ventricular ejection fraction, lower creatinine clearance, higher anatomical SS, and younger age were shown to be positive factors indicating coronary bypass $[6,12]$.

Hayiroglu et al. [17] reported that SS-II provides a prognostic value for in-hospital mortality and MACE in STEMI patients who had complications related to cardiogenic shock. This result indicates that SS-II is predictive not only for long-term outcomes but also for in-hospital outcomes of STEMI patients. In addition, SS-II is superior to SS in the prediction of in-hospital mortality in patients with STEMI complicated with cardiogenic shock treated with primary PCI. Hayiroglu et al. [17] showed that SS-II is not favorable for predicting in-hospital target lesion revascularization, stent thrombosis, or recurrent myocardial infarction. Our study, however, focused on NSTEMI patients and showed that SS-II has a prognostic value for in-hospital mortality and MACE in NSTEMI patients treated with primary PCI. In contrast to the findings of the previous study, in our study SS-II was also able to predict in-hospital mortality, nonfatal myocardial infarction, and stent thrombosis. We did, however, exclude patients with shock from the in-hospital target lesion revascularization, stent thrombosis, and recurrent myocardial infarction results.

Song et al. [18] demonstrated the ability of SS-II to predict 2-year mortality in a population of patients with complex CAD undergoing PCI and concluded that SS-II is better than SS at predicting 2-year mortality for patients with complex CAD undergoing PCI. Salvatore et al. [19] revealed that SS-II might be a useful tool for predicting the risk of adverse clinical events in patients with ACS and severe CAD undergoing PCI at a 1-year follow-up. 
The SS-II score used in these 2 studies was predictive of the events at the 1- and 2-year follow-ups. As our study showed, SS-II is effective in determining in-hospital adverse events. A review of the relevant literature reveals that no previous studies have compared SS-II to other risk scoring systems designed to assess clinical risk and the extent of CAD [20-22]. In this study, we first compared 4 scoring systems. Although the SS-II score has similar clinical outcomes to the other scores, it is better able to identify inhospital events. The limitations of this study included its single-center, retrospective nature, the small number of patients, and the fact that it did not assess SS-II changes that might have occurred during long-term follow-ups (such as changes in creatinine clearance or left ventricular ejection fraction). In addition, the association between changes in SS-II and the presence of major adverse events during the long-term follow-up period were not assessed.

\section{Conclusion}

As a risk score that combines both anatomical and clinical variables, SS-II, TIMI, and GRACE risk scores are useful in the prediction and risk stratification of in-hospital MACE; however, of the studied methods, SS-II is the most useful tool in assessing overall in-hospital adverse events.

\section{References}

1 Antman EM, Cohen M, Bernink PJ, et al: The TIMI risk score for unstable angina/non-ST elevation MI: a method for prognostication and therapeutic decision making. JAMA 2000;284:835-842.

2 Kurtul S, Sarli B, Baktir AO, et al: Neutrophil to lymphocyte ratio predicts SYNTAX score in patients with non-ST segment elevation myocardial infarction. Int Heart J 2015;56: $18-21$

3 Erkan H, Kırış G, Korkmaz L, et al: Relationship between nitrate-induced headache and coronary artery lesion complexity. Med Princ Pract 2015;24:560-564.

4 Yan AT, Yan RT, Tan M, et al: Risk scores for risk stratification in acute coronary syndromes: useful but simpler is not necessarily better. Eur Heart J 2007;28:1072-1078.

5 Granger CB, Goldberg RJ, Dabbous O, et al: Predictors of hospital mortality in the global registry of acute coronary events. Arch Intern Med 2003;163:2345-2353.

6 Farooq V, van Klaveren D, Steyerberg EW, et al: Anatomical and clinical characteristics to guide decision making between coronary artery bypass surgery and percutaneous coronary intervention for individual patients: development and validation of SYNTAX score II. Lancet 2013;381:639-650.

$7 \mathrm{Xu}$ B, Généreux P, Yang Y, et al: Validation and comparison of the long-term prognostic capability of the SYNTAX score-II among 1,528 consecutive patients who underwent left main percutaneous coronary intervention. JACC Cardiovasc Interv 2014;7:11281137.

8 Campos CM, van Klaveren D, Iqbal J, et al: Predictive performance of SYNTAX Score II in patients with left main and multivessel coronary artery disease. Circ J 2014;78:19421949 .
9 Farooq V, Girasis C, Magro M, et al: The CABG SYNTAX Score - an angiographic tool to grade the complexity of coronary disease following coronary artery bypass graft surgery: from the SYNTAX Left Main Angiographic (SYNTAX-LE MANS) substudy. EuroIntervention 2013;8:1277-1285.

10 Tang EW, Wong C-K, Herbison P: Global Registry of Acute Coronary Events (GRACE) hospital discharge risk score accurately predicts long-term mortality post acute coronary syndrome. Am Heart J 2007;153:29-35.

11 Bekler A, Altun B, Gazi E, et al: Comparison of the GRACE risk score and the TIMI risk index in predicting the extent and severity of coronary artery disease in patients with acute coronary syndrome. Anatol J Cardiol 2015; 15:801-806

12 Farooq V, Serruys PW, Bourantas C, et al: Incidence and multivariable correlates of longterm mortality in patients treated with surgical or percutaneous revascularization in the synergy between percutaneous coronary intervention with taxus and cardiac surgery (SYNTAX) trial. Eur Heart J 2012;33:31053113.

13 Singh M, Rihal CS, Gersh BJ, et al: Twentyfive-year trends in inhospital and long-term outcome after percutaneous coronary intervention: a single-institution experience. Circulation 2007;115:2835-2841.

14 Yadav M, Palmerini T, Caixeta A et al: Prediction of coronary risk by SYNTAX and derived scores: synergy between percutaneous coronary intervention with taxus and cardiac surgery. J Am Coll Cardiol 2013;62:1219-1230.

15 Chieffo A, Meliga E, Latib A, et al: Drug-eluting stent for left main coronary artery disease: the DELTA registry: a multicenter registry evaluating percutaneous coronary intervention versus coronary artery bypass grafting for left main treatment. JACC Cardiovasc Interv 2012;5:718-727.
16 Girasis C, Garg S, Räber L, Sarno G, et al SYNTAX score and Clinical SYNTAX score as predictors of very long-term clinical outcomes in patients undergoing percutaneous coronary interventions: a substudy of SIRolimus-eluting stent compared with pacliTAXel-eluting stent for coronary revascularization (SIRTAX) trial. Eur Heart J 2011;32:31153127.

17 Hayiroglu MI, Keskin M, Uzun AO, et al: Predictive value of SYNTAX score II for clinical outcomes in cardiogenic shock underwent primary percutaneous coronary intervention: a pilot study. Int J Cardiovasc Imaging 2018; 34:329-336.

18 Song Y, Gao Z, Tang X, et al: Usefulness of the SYNTAX score II to validate 2 -year outcomes in patients with complex coronary artery disease undergoing percutaneous coronary intervention: a large single-center study. Catheter Cardiovasc Interv DOI: $10.1002 / \mathrm{ccd}$. 27321.

19 Salvatore A, Boukhris M, Giubilato S, et al Usefulness of SYNTAX score II in complex percutaneous coronary interventions in the setting of acute coronary syndrome. J Saudi Heart Assoc 2016;28:63-72.

20 Ellis SG, Vandormael MG, Cowley MJ, et al: Coronary morphologic and clinical determinants of procedural outcome with angioplasty for multivessel coronary disease: implications for patient selection. Multivessel Angioplasty Prognosis Study Group. Circulation 1990;82: 1193-1202.

21 Hamburger JN, Serruys PW, Scabra-Gomes $\mathrm{R}$, et al: Recanalization of total coronary occlusions using a laser guidewire (the European TOTAL Surveillance Study). Am J Cardiol 1997;80:1419-1423.

22 Lefèvre T, Louvard Y, Morice MC, et al: Stenting of bifurcation lesions: classification, treatments, and results. Cathet Cardiovasc Diagn 2000;49:274-283.
The Predictive Role of SYNTAX Score II in Adverse Cardiovascular Events
Med Princ Pract 2018;27:459-465 